\title{
Plasma Electric Field Measurements as a Diagnostic of Neutral Sheets in Prominences
}

\section{P. Foukal}

CRI, Inc., Cambridge, MA 02139, USA E-mail: pfoukal@world.std.com

\begin{abstract}
.
Measurements of plasma electric fields offer, in principle, a direct test for the presence of neutral sheets in prominences. Macroscopic electric field intensities of order $1-10 \mathrm{~V} \mathrm{~cm}^{-1}$ are an essential element of MHD models of prominences containing neutral sheets. These fields should be detectable with our electrograph techniques using the high Paschen-series lines in the NIR, and the 15-9 and 16-9 transitions of $H I$ around $10.5 \mu$. We discuss the upper limits of $1 \mathrm{~V} \mathrm{~cm}^{-1}$ we have achieved so far, and their implication for our ability to distinguish prominence models of the idealMHD type (e.g., Kippenhahn-Schluter 1957), from those fundamentally different models (e.g., Kuperus and Tandberg-Hanssen 1967, Martens and Kuin 1989) in which neutral sheets play a central role.
\end{abstract}

\section{Introduction}

Ideal MHD models of prominence support (e.g., Kippenhahn and Schluter 1957) predict very small $\mathrm{E}\left(\leq 10^{-8} \mathrm{~V} \mathrm{~cm}^{-1}\right)$ for classical or even anomalous resistivity values. Such values of $\mathrm{E}$ would be completely undetectable. Current sheet models (e.g., Kuperus and Tandberg-Hanssen 1967, Martens and Kuin 1989) predict a very large drop along the current sheet $\left(\geq 10^{8} \mathrm{~V}\right)$. An electric field of order $1-10 \mathrm{~V} \mathrm{~cm}^{-1}$ should be detectable either i) within the prominence (i.e., around the current sheet); or ii) within the resistance of the cool legs of the prominence; or iii) in the upper photosphere, where the circuit should be completed (Foukal and Behr 1995). Table 1 summarizes previous electric field measurements in prominences.

\section{Ongoing Electrograph Observations}

The 16-9 transition of $\mathrm{H} \mathrm{I}$ at $925.6 \mathrm{~cm}^{-1}(10.8 \mu)$ was identified recently in our Fourier transform spectrometer (FTS) spectra of quiescent prominences by E. Chang (1996, private communication). One of our FTS spectra is shown in Figure 1, with this line marked. Calculations by R. Casini (Figure 2) show the sensitivity $\left(\mathrm{m} \AA / \mathrm{V} \mathrm{cm}^{-1}\right)$ of this line to electric fields directed perpendicular and parallel to the prominence magnetic field. Our first FTS spectra polarimetry on this line (Figure 3) indicates a sensitivity of about $1-2 \mathrm{~V} \mathrm{~cm}^{-1}$ should be achievable on bright quiescent prominences. Future observing runs at Kitt Peak will focus on electric fields in the prominence body and legs. 
Table 1. Previous Electric Field Measurements in Prominences

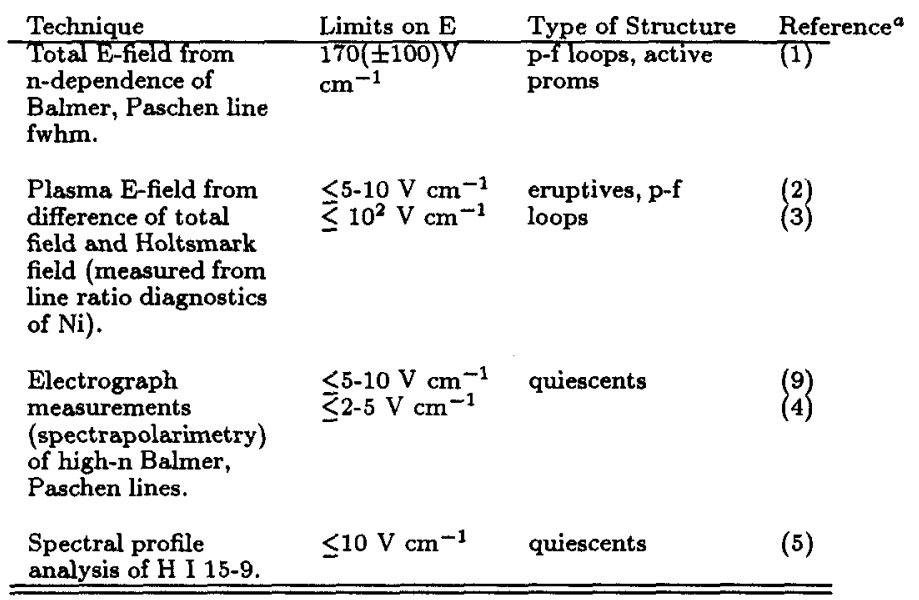

${ }^{a}$ numbers refer to ordering in the Reference section of this paper.

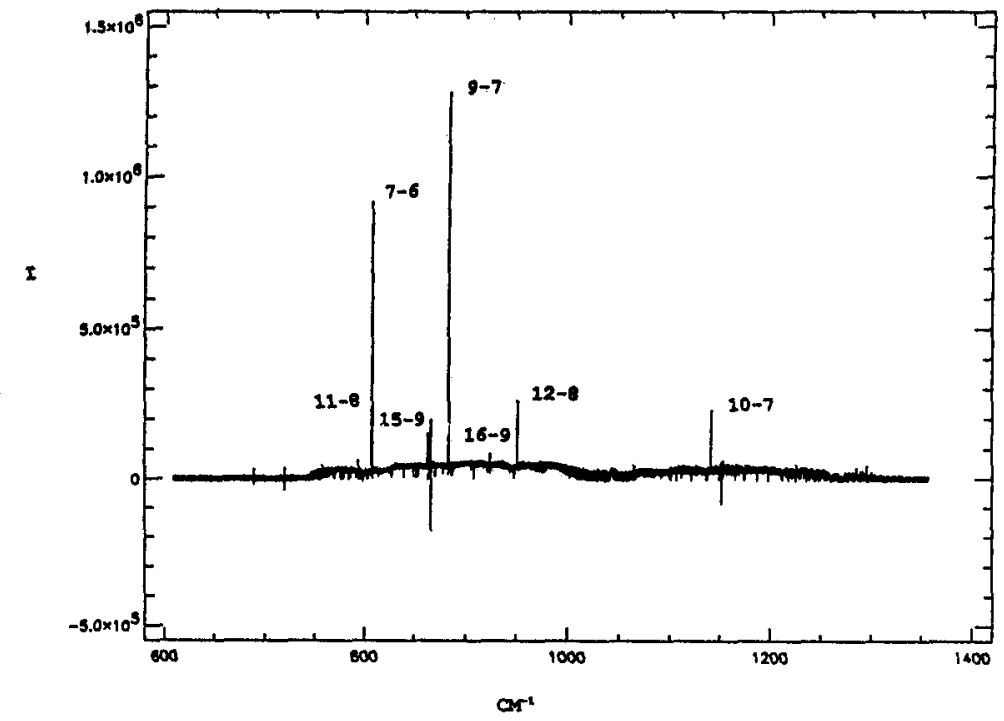

Figure 1. Spectrum of a quiescent prominence, over the wavenumber range $600-1400 \mathrm{~cm}^{-1}$, obtained with the FTS at the McMath telescope, Kitt Peak. 

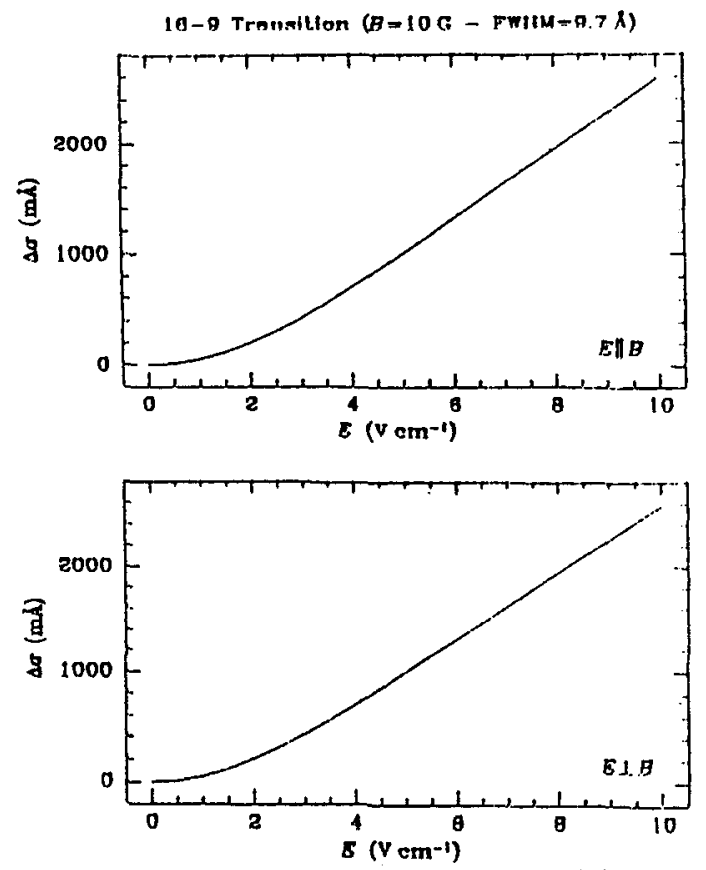

Figure 2. Plots of the difference in full-width half-maximum of the H I 16-9 transition, when only $\pi$ and only $\delta$-components of the Stark profile are observed. The ordinate is line width modulation in $m \AA$; the abcissa is electric field intensity in $\mathrm{V} \mathrm{cm}^{-1}$.

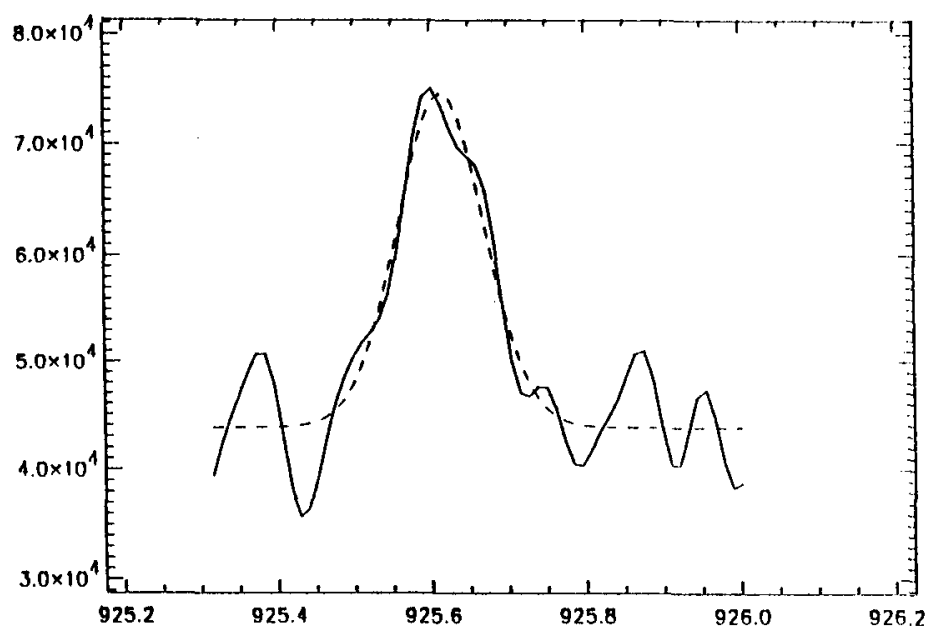

Figure 3. Line profile of H I 16-9 observed (-) and gaussian fitted (-). FTS spectrapolarimetry on April 15, 1997. 
Electric fields associated with completion of the prominence circuit through the photosphere might be measurable using a Stark-sensitive photospheric absorption line. The most interesting candidate is the H I Paschen transition 7-3 at $\lambda$ 10049. We are collaborating with $\mathrm{R}$. Casini in estimating the Stark sensitivity of this absorption line.

\section{Conclusions and Future Directions}

1. Spectrapolarimetry using Balmer and Paschen series lines has so far revealed no plasma electric fields in quiescent prominences, at a sensitivity level reaching 2-5 $\mathrm{V} \mathrm{cm}^{-1}$, which is comparable to the electric fields expected in the body and/or legs of the prominence if an extended neutral sheet is present. Ongoing FTS spectrapolarimetry using the highly Starksensitive 16-9 transition should yield sensitivity of $1-2 \mathrm{~V} \mathrm{~cm}^{-1}$, in the body and legs of these structures.

2. Spectrapolarimetry using the Paschen 7-3 photospheric absorption line may enable us to determine if a significant part of the "missing potential drop" in a neutral-sheet associated prominence, might actually occur in the photospheric portion of the prominence's equivalent circuit.

3. Detection of electric fields within the prominence body, legs, or associated photosphere would provide a unique test of neutral sheet existence in the solar atmosphere. Absence of detectable $\mathrm{E}$ would call into question neutral sheet models of quiescent prominences. However, it is unlikely that even the sensitivity increase achieved with the 16-9 line will be sufficient to detect the (model-independent) motional electric field due to ionized particles collisionally crossing the magnetic field lines. This anisotropic, microscopic electric field must be present in the prominence plasma at the $0.5 \mathrm{~V} \mathrm{~cm}^{-1}$ level (Moran and Foukal 1991).

Acknowledgments. This work is supported by the Solar-Terrestrial Program of the Atmospheric Sciences Division, under NSF grant ATM 9301832.

\section{References}

Foukal, P., Miller, P., and Gilliam, L. 1983, Solar Phys. 83, 83

Foukal, P., Hoyt, C., and Gilliam, L. 1986, ApJ, 303, 861

Foukal, P., Little, R., and Gilliam, L. 1988, Solar Phys. 114, 65

Foukal, P. and Behr, B. 1995, Solar Phys. 156, 293

Foukal, P. and Casini, R. 1996, BAAS, 28, 876

Kippenhahn, R. and Schlüter, A. 1957, Z. f. Astrophys., 43, 36

Kuperus, M. and Tandberg-Hanssen, E. 1967, Solar Phys., 2, 39

Martens, P.C.H. and Kuin, N.P.M. 1989, Solar Phys., 122, 263

Moran, T. and Foukal, P. 1991, Solar Phys. 135, 179 